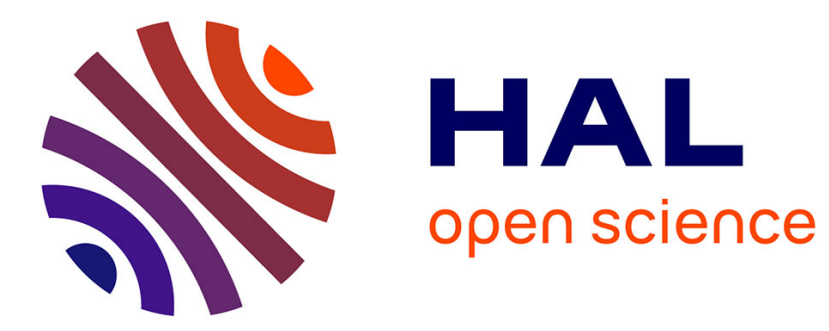

\title{
Le décor, ou l'art de tourner en rond.
}

\author{
Anne Larue, Ségolène Le Men
}

\section{To cite this version:}

Anne Larue, Ségolène Le Men. Le décor, ou l'art de tourner en rond. : L'ornement dans l'illustration romantique. Romantisme: la revue du dix-neuvième siècle, 1992, Le conte et l'image (78), p. 61-74. 10.3406/roman.1992.6078 . hal-00607723

\section{HAL Id: hal-00607723 \\ https://hal.science/hal-00607723}

Submitted on 11 Jul 2011

HAL is a multi-disciplinary open access archive for the deposit and dissemination of scientific research documents, whether they are published or not. The documents may come from teaching and research institutions in France or abroad, or from public or private research centers.
L'archive ouverte pluridisciplinaire HAL, est destinée au dépôt et à la diffusion de documents scientifiques de niveau recherche, publiés ou non, émanant des établissements d'enseignement et de recherche français ou étrangers, des laboratoires publics ou privés. 
Anne LARUE

Ségolène $L E M E N$

Le décor, ou l'art de tourner en rond

L'ornement dans l'illustration romantique

Toute l'histoire de l'illustration est fondée sur une grande opposition entre le décoratif et l'illustratif, qui recoupe un système hiérarchisé d'emplacements, distinguant planches hors-texte et ornements dans le texte. Parmi les ornements, les bandeaux (liminaires) et les culs-de-lampe (terminatifs) conjuguent leur vocation décorative à une fonction de subdivision du texte et du livre. Les illustrations du cours du texte, qu'elles soient planches ou, à certaines époques, vignettes, sont régies par un rapport sémantique entre le texte et l'image, parfois précis, parfois beaucoup plus diffus.

Dans le livre illustré romantique, cette hiérarchie des espaces, si elle subsiste, se trouve cependant remise en cause ; les catégories du décoratif et de l'illustratif se perpétuent tout en se dissolvant, grâce à l'invention très particulière de la vignette. Aux "sujets"1 dans le texte ou hors texte, revient le rôle de ponctuer la narration ; à l'ornement, le droit d'y faire allusion, mais aussi la liberté de s'en dispenser. Propre au romantisme est cette façon de maintenir les anciennes catégories et leurs registres traditionnels, tout en les bouleversant de fond en comble par un effet de contamination réciproque. Bien qu'elle soit particulièrement sensible dans le frontispice, l'interférence des catégories se manifeste dans tous les espaces figurés, jusques et y compris dans celui du bandeau, qu'aborde cet article.

Ambigu par nature, le frontispice est en fin de compte moins révélateur, en raison de la complexité des critères qu'exige son analyse. Le cas du bandeau, en revanche, est très net : décoratif à l'origine, il glisse dans le registre illustratif d'une manière clairement repérable. Ainsi révèle-t-il le

${ }^{1}$ Le mot "sujet" est utilisé dans le vocabulaire de l'édition illustrée pour désigner les dessins, motifs figurés de divers formats, commandés par l'éditeur à l'illustrateur en vue de la gravure sur bois. C'est ainsi qu'il apparaît fréquemment dans les lettres de commande. 
travail de cette porosité, de cette fusion des espaces décoratifs et illustratifs qui semble être le propre du romantisme dans le domaine qui nous occupe.

Vignette contre bandeau ? Le duel n'est pas si simple. L'articulation de ces deux espaces, qui met en jeu la structure même du livre romantique illustré, aurait-elle une influence sur les métaphores qui président à cette structure, consacrant l'interférence de l'architectonique et du végétal ?

A travers travers l'édition Bourdin des Mille et une Nuits ${ }^{2}$, nous allons voir comment le texte, d'une part, et le livre lui-même, d'autre part, manifestent la révolution illustrative de la vignette.

\section{Le texte : ornement et illustration}

Dans ce livre romantique comme dans tant d'autres, la présence de l'ornement est très marquée : bandeaux et cul-de-lampe rythment la succession des Mille et une Nuits. Chacun se souvient comment Scheherazade, mariée au sultan qui chaque matin tue son épouse de la nuit, diffère par ses contes habilement interrompus sa propre mise à mort, chaque matin reportée. C'est l'essence même du suspense que la tradition des Mille et une Nuits, contes arabes traduits en $1704^{3}$ par Galland, propose aux lecteurs français ${ }^{4}$. Pour l'éditeur romantique Ernest Bourdin, ce livre par nature morcelé se prête tant à la coupure de la livraison qu'à l'abondance de gravures - ornements et illustrations. Il répond d'emblée, par la nature même de la narration qui le sous-tend, à l'attente du public élargi des "nouveaux lecteurs", pour lesquels l'image dans le texte est tout à la fois un repère, une nécessaire pause de lecture, et un embellissement.

L'ornement répond à cette dernière fonction. Il est peu soumis au texte : on ne saurait lui reprocher d'être non conforme à la narration, alors qu'on attend de l'illustration "dans le texte" une parfaite adéquation avec ce qui est

2 Les Mille et une Nuits, contes arabes traduits par Galland. Edition illustrée par les meilleurs artistes français et étrangers, revue et corrigée sur l'édition princeps de1704, augmentée d'une dissertation sur les Mille et une Nuits, par M. le baron Sylvestre de Sacy... Ernest Bourdin et Cie., s. d . (1838-1840), 3 vol. gr. in $8^{\circ}$.

${ }^{3}$ Le système de vente du livre par livraisons, comparables aux livraisons de la presse, caractérise le livre illustré romantique français, depuis le célèbre Gil Blas illustré par Gigoux (Paulin, 1835).

${ }^{4}$ Voir à ce sujet notre commun article "Un feuilleton à suspense : les Mille et une Nuits", La Licorne, 1992 (sous presse). 
écrit ${ }^{5}$. Le même lecteur qui sera gêné par une vignette "réaliste" mais mal venue, aura de l'indulgence envers bandeaux et culs-de-lampe, admettant des motifs sans rapport avec le texte - Walkyrie à cheval sur un oiseau-roc ${ }^{6}$ - ou frisant la fantaisie de l'absurde à partir de motifs Rococo proches de la poésie alexandrine - "putti" jouant avec raquettes et balles/bulles de savon autour d'un jet d'eau? (fig.1).

Le lieu où se situe l'illustration dans le livre induit une attente de la part du lecteur, qui ne cherche pas n'importe où n'importe quelle illustration. Dans le cours du texte, prennent place des illustrations comparables à des images de mémoire ${ }^{8}$, qui récapitulent des unités textuelles d'extension variable ; autour de ces images, l'espace du livre illustré développe volontiers la métaphore d'une architecture parcourue peu à peu, depuis le seuil du frontispice, qui détermine l'abord du livre entier, jusqu'aux chambres de mémoire traversées l'une après l'autre, et dont chacune s'ouvre sur un bandeau et se clôt sur un cul-de-lampe. Cette métaphorisation globale procède par

5 Vignette dans le texte, p. 243, tome I. L'exemple du réemploi d'une gravure narrative dans une édition ultérieure des Nuits de Bourdin (édition Garnier-Frères, 1870) le montre clairement. Dans le premier tirage du bois (Bourdin), la gravure illustre le mariage de Zobéïde avec un très beau et très vertueux jeune homme. Une femme frappe dans ses mains - geste représenté par le personnage de droite. Lors du réemploi (Garnier), la même gravure illustre cette fois un épisode licencieux du récit-cadre, celui où la sultane infidèle frappe dans ses mains pour appeler son amant noir, Masoud, tandis que suivantes et esclaves noirs se livrent à la luxure. Le réemploi est ingénieux, puisque le geste de la sultane est le même que celui de la femme convoquant la noce - à cette exception près que le puissant esclave noir qui s'approche de la suivante se trouve être, réemploi oblige, un fin jeune moustachu coiffé d'un chapeau à aigrette, ce qui nuit à la crédibilité du récit et met le lecteur est en droit de se sentir légitimement frustré. Le procédé, courant, du réemploi rend l'illustration dans le texte un peu moins littérale.

${ }^{6}$ Cul-de-lampe p. 114, t. I. Ce personnage de chasseresse armée d'un arc n'apparait pas dans le texte. L'oiseau-roc est un animal extraordinaire, l'un des plus fameux du bestiaire des Mille et une Nuits. Il est d'une grandeur prodigieuse et d'une méchanceté cruelle. Des héros habiles - comme Sindbad - savent néanmoins s'accrocher à l'oiseau-roc à son insu, pour être transporté, par la voie des airs, le plus loin possible de lieux inhospitaliers.

${ }^{7}$ Bandeau p. 200, t. I. Tout est prétexte à rondeurs et enroulements dans ce gracieux et aérien bandeau, où les petits personnages sont littéralement portés par les volutes de feuilles d'acanthe opportunément glissés sous leurs pieds. Balles volantes, coeurs de rosaces, grains de raisin, sexe des anges : le même motif de petite boule ronde scande - avec un soupçon d'impertinence dans le détail, mais quel censeur s'arrête à de si petites choses ? - cette fine gravure aux deux garçons nus. L'art de l'image serait-il celui de tout montrer sans que rien ne se voie?

8 Voir Frances Yates, L'art de la mémoire, Gallimard, 1975 (éd. or. angl. 1966). 
figure étymologique, puisqu'elle appartient au sens premier de chacun des mots du lexique de l'illustration, issus du vocabulaire de l'architecture : ainsi, en termes d'architecture, le "bandeau" est une "moulure pleine à saillie rectangulaire dont la largeur est sensiblement égale à la saillie", et le "cul-delampe" un "organe en surplomb portant une charge, formé de plusieurs assises appareillées en cône, en pyramide renversée ou en quart de sphère"9. L'édition Perrotin de Notre-Dame de Paris (1844) ordonne toute son illustration gothique autour de la métaphore architectonique du livrecathédrale qui se déploie dès le frontispice, et qui, de surcroît, appartient profondément à la conception hugolienne du roman. Quelques années auparavant, dans l'édition Bourdin des Mille et une Nuits, le modèle architectural primait déjà, accommodé à la sauce orientale : en fronton, le frontispice à l'architecture compartimentée annonce le "programme" du livre ; puis, en arc à double accolade (ou en arche, parfois), des bandeaux "ouvrent" chacun des chapitres - ici, ce sont des "nuits" -, tandis que des culs-de-lampe viennent clore et supporter symboliquement les masses d'écriture et les blocs typographiques. Le texte est calibré, encadré et rythmé par ces illustrations d'une nature particulière, qui relèvent moins de la mise en texte que de la mise en livre et en imprimé, considérée comme une forme de construction. La lecture est alors identifiée à une mnémotechnique, qui use des vieilles recettes rhétoriques des arts de mémoire. Cependant, au fil du texte, s'insèrent des vignettes "littérales" fortement rattachées à la narration, dont le "grand sujet" hors-texte est - si l'éditeur en a les moyens ${ }^{10}$ - un luxueux avatar ${ }^{11}$, propre aux éditions dites "keepsake" et aux ouvrages "de luxe à bon marché" vendus par livraisons illustrées.

9 J.-M. Pérouse de Montclos (dir.), Principes d'analyse scientifique. Architecture. Méthode et vocabulaire, Imprimerie nationale, 1972, p. 192 (bandeau) et 122 (cul-de-lampe).

10 Car la planche hors-texte ou "grand sujet" est payée plus cher (au dessinateur comme au graveur) que les "petits sujets" (voir note 1).

11 Sans légende dans l'édition Bourdin qui nous occupe, pour des raisons essentiellement techniques, la gravure hors-texte, dans les retirages postérieurs (Garnier, 1870, par exemple), s'accompagne d'une phrase tirée du texte qui la situe précisément et la rattache à la narration. Manifestement, si le bandeau et le cul-de-lampe appartiennent au registre décoratif, les illustrations "hors-texte", tout comme celles qui ponctuent le fil du texte, sont de nature narrative. Cette fonction sera soulignée, dès que la technique le permettra, par l'ajout d'une légende précisant à quel passage se réfère précisément l'image (en particulier dans les éditions populaires illustrées) ; dès la toute première édition illustrée des Nuits, celle du Cabinet des Fées (1785), l'usage de l'illustration hors-texte s'accompagnait du rappel très précis d'une phrase du texte faisant office de légende. 
En raison de leur lien étroit avec le texte, les vignettes au fil du texte et les planches hors-texte constituent ainsi les "illustrations narratives", par opposition aux lieux de l'ornement et de l'édification du livre, essentiellement bandeaux et culs-de-lampe. La situation, liminaire, progressive ou terminative, de l'illustration, par rapport aux masses de texte, détermine donc d'emblée son statut.

\section{Style romantique et style classique : cadre et vignette}

Le style classique serait-il rectangulaire et cadré, par opposition au style rond et centré, romantique ? N'importe quel tableau de David, avec son pavage en perspective, la ponctuation rythmique de sa toile de fond architecturale, la mise en scène efficace de ses figures centrales, confirme la première partie de cette hypothèse. Pourrait-on dire que le romantique est en tant que branche de ce baroque éternel en faveur duquel argumente Eugenio d'Ors ${ }^{12}$ - au contraire rond, tournoyant, excentré, périphérique ? Si Delacroix note, dans son journal, que "l'influence des lignes principales est immense dans une composition", nul doute que ce soit pour privilégier "un mouvement, une variété et en même temps une unité incomparables" : celle de Rubens dans sa Chasse à l'Hippopotame ${ }^{13}$. Le peintre est fort loin de haïr le mouvement qui déplace les lignes. Femmes renversées, figures ployantes, le romantisme de Delacroix est courbe, incontestablement.

Cette grande opposition stylistique, par laquelle Wölfflin délimite style classique et style baroque, se prolonge, dans l'histoire du livre illustré, par le passage du livre de conception néo-classique au livre romantique : les éditions néo-classiques publiées par Didot présentent quelques rares planches hors-texte rectangulaires, comparables à des tableaux, "fenêtres" s'ouvrant dans le livre, tandis que, dans le livre romantique, les vignettes, aux contours flottants, de forme grossièrement circulaire, semblent rayonner autour de leur noyau et se multiplient dans le texte. Forme centrée et rayonnante, la vignette est par définition sans marge, sans cadre ${ }^{14}$.

12 Eugenio d'Ors, Du Baroque, trad. franç. 1968 (1935). Le baroque ne serait pas un style daté mais une inclination générale de l'esprit humain, une "constante historique" (p. 78) décelable à toutes les époques.

${ }^{13}$ E. Delacroix, Journal, 25 janvier 1847.

${ }^{14}$ Ce développement s'inspire de l' analyse menée par Henri Zerner et Charles Rosen dans Romanticism and Realism. The mythology of nineteenth Century Art, New York, Viking Press, 1984 (trad. française Albin michel, 1986). 
L'opposition stylistique entre romantisme et classicisme s'inscrit donc, pardelà l'histoire des formes, jusque dans l'histoire du livre illustré : c'est la conception de l'image même qui manifeste ces ruptures stylistiques, par l'invention de la vignette.

Les motifs eux-mêmes participent de cette grande opposition stylistique : ainsi, la vignette représentant Scheherazade et Dinarzade (fig. 2) formant un couple alangui sur un sofa, multiplie les figures du cercle et du rayonnement autour de motifs tels que perles, turbans, médaillon, coussins, arceau. Le répertoire de l'ornementation des tissus et du décor intérieur souligne le parti-pris de l'arabesque. Ce goût des volutes, qui se manifeste aussi dans la manière du dessin et dans le traitement du corps féminin, n'est pas sans rappeler la fameuse "méthode des oeufs" que Gigoux prétend tenir de Delacroix ${ }^{15}$. N'en dirait-on pas autant de la danse mouvante des cimeterres ${ }^{16}$, des turbans, des poufs rayonnants, des plumes de paon, du répertoire de motifs issu du bric-à-brac oriental, qui illustre à tout instant les Mille et une Nuits ? Le prétexte du bazar ne trompe personne : ces motifs ne sont tous là - comme les paons eux-mêmes - que pour faire la roue. Le mot de "vignette" invite d'emblée à penser la spirale comme figure fondatrice de ce nouveau langage des formes. Vignette vient en effet de ..."vigne", comme son nom l'indique, par allusion à un motif en rinceau, dérivé de la feuille d'acanthe et lové dans le même enroulement, mais figurant les vrilles de la vigne (fig.1). Ce motif figurait souvent dans les marges des manuscrits du gothique fantastique, en compagnie d'autres sujets de fantaisie - petits personnages, fleurs ou fruits - choisis pour leurs qualités décoratives, mais n'excluant pas la liberté du caprice. Blake, l'artiste préromantique anglais, se plut à agrémenter ainsi les bouts de ligne, marges, hampes de lettrines dans les livres qu'il écrivait, illustrait, gravait, imprimait, et finalement mettait en vente, réunissant à lui seul toute la chaîne des acteurs impliqués dans l'édition d'un livre. Par la suite, dans le livre illustré romantique, les jeux de figure étymologique sur "vignevignette" se retrouvent constamment, au point de devenir presque un cliché iconographique du vocabulaire de l'ornement d'illustration.

\section{De l'ordre architectural à la croissance végétale}

15 Gigoux, Causeries avec les artistes de mon temps, Calmann-Lévy, 1885. 16 Voir la vignette p. 132, illustrant le conte des Dames de Bagdad : "aussitôt une porte s'ouvrit, et sept esclaves noirs, puissants et robustes, entrèrent le sabre à la main"... L'ensemble du dispositif de la vignette indique un mouvement de spirale tournoyante et de formes concentriques. 
Comme une décalcomanie, la vignette va de support en support, se déplace dans tous les espaces du livre, bouleverse l'ordre établi, fait éclater, par sa mobilité native, la noble et raide architecture qui préside au livremonument. Au livre architectural se substitue ainsi le livre-plante, fondé sur un modèle organique plus proche par ailleurs de l'esprit romantique. Le livre pousse comme le luxuriant végétal par excellence : l'arbre de la connaissance.

Le modèle tectonique d'esprit plus "classique" - et son corollaire, la mise en scène théâtrale, sous-ensemble de la catégorie de l'architecture dans la perspective qui nous occupe ici - n'en disparaissent pas pour autant ; mais du moins sont-ils fortement atténués par l'irruption de la vignette narrative, élément perturbateur par excellence, autonome et mobile. Combat des pierres contre le végétal ? Le registre ornemental de l'illustration - principalement bandeaux et cul-de-lampe - reste étroitement soumis à l'architecture. Le bandeau reste un arc, sous lequel le lecteur est invité à passer pour accéder au texte. Un arc, justement : ne dirait-on pas que dans la forme essentiellement rectangle du bandeau, frise rythmée et symétrique marquant le début de chapitre, la forme courbe s'ingénie discrètement à entrer ? Ainsi se manifeste l'essence profondément subversive de l'image, apparemment soumise à l'ordre du texte qui, dans un livre, occupe le centre de l'espace, mais profitant des lisières, des marges et des coins, pour révéler à l'observateur attentif un luxe de détails qui ne sont pas gratuits. C'est, par exemple, dans la sphère de l'ornement et du bandeau que glisse, accessible à qui le cherche, le détail licencieux sur lequel se fonde l'érotisme voilé des Nuits, dans l'édition Bourdin : ainsi, le bandeau des "putti" aux raquettes (fig.1), ou encore celui des orientaux à la grenade, où abondent figures de la fécondité (grenade, oeufs) et de la castration (sabres menaçants) dans une composition symétrique dont la partie centrale est saturée de symboles sexuels à peine dissimulés par les volutes de l'ornement (fig.3).

Non contente d'avoir droit de cité dans l'illustration narrative, qui est son triomphe, la vignette, parée de toutes les séductions de la "scène de genre" et de toute l'efficacité de la publicité17, ne tarde pas à occuper le terrain réservé au décoratif, brouillant l'ordre et les lieux de l'architecture sous-jacente.

\footnotetext{
${ }^{17}$ Les livres étaient plus souvent connus par la vignette de leur prospectus de vente et de la couverture illustrée, dont l'affiche de librairie agrandissait le motif, que par leur contenu, et certains ne doivent même leur célébrité qu'à une vignette de couverture à laquelle ils s'identifient, comme le remarquait Champfleury dans Les Vignettes romantiques (Dentu, 1883).
} 
Toujours différente, souple d'emploi ${ }^{18}$, la vignette ne dépare ni un bandeau, ni un cul-de-lampe ; il n'est pas de lieu, dans le livre, qui se donne le droit de la refuser. Deux gravures illustrant les voyages de Sindbad le marin, à une page $\mathrm{d}^{\prime}$ intervalle ${ }^{19}$, traduisent ce caractère interchangeable : la première, en cul-delampe, montre une main monstrueuse se saisissant d'un petit homme, la seconde, dans le texte, montre un autre petit homme, identique au premier, dans la gueule géante d'un serpent. La situation narrative ${ }^{20}$, le traitement de l'image, le jeu de gigantisme, la (petite) taille du motif, tout est similaire d'une gravure à l'autre : grâce à sa petite taille, la vignette narrative a pris possession d'un lieu traditionnellement dévolu à l'ornement, celui du cul-delampe ; inversement, le cul-de-lampe est susceptible de devenir une petite vignette dans le texte.

Un autre exemple de circulation des images, résultant des qualités d'adaptation et de mobilité de la vignette, est le déplacement d'un motif de cul-de-lampe en bandeau : représentant "les pabouches (sic) et la cognée" qui trahiront le deuxième Calender ${ }^{21}$, le bandeau, ici, renonce au motif de l'arche et au primat de la symétrie : il adopte la morphologie traditionnelle du cul-delampe ! C'est ainsi qu'il regroupe, à la manière des symboles ou des trophées ornementaux, quelques objets significatifs ${ }^{22}$. Mais ce déplacement en position de bandeau - un bandeau qui renie ici les lois de son genre - n'empêche pas l'image d'être avant tout - et quelle que soit sa position - une vignette, fortement impliquée dans la narration : en effet, c'est à ces babouches et à

18 C'est-à-dire techniquement reproductible en différents endroits du livre, les techniques de la gravure permettent la circulation des motifs.

${ }^{19}$ p. 347 et 349 , t. II.

20 Tous les compagnons de Sindbad disparaissent, vaincus par des monstres divers et successifs : d'abord un cyclope anthropophage que Sindbad et ses compagnons aveuglent dans la plus pure tradition de l'Odyssée, puis un serpent tout aussi géant et affamé. L'unité de grandeur du gigantisme est, dans le texte de Galland, le palmier : le géant est "de la hauteur d'un grand palmier" (p. 344), le serpent est "long comme un palmier" (p. 349).

21 "Calenders" est le nom donné aux membres d'une classe de derviches mendiants et vagabonds, au XIIIème siècle surtout, qu'on rencontrait de l'Asie centrale au Maroc. Musulman quelque peu picaresque ou "hippy" avant l'heure, parfois dresseur de singes ou convoyeur, le calender est un type tout trouvé pour les Mille et une nuits. Dans le conte des Dames de Bagdad, dont il est question ici, les trois calenders sont porteurs de cette connotation d'aventure, de non-conformisme et de marginalité, festoyant avec des dames de rencontre à qui ils racontent leur fortune et ses revers.

${ }^{22}$ Le groupement d'objets en un tableautin à valeur symbolique ou allusive, motif fort prisé dans le cul-de-lampe, n'est pas sans rappeler les "trophées" de la tradition décorative, représentant par des objets spécifiques les prises de guerre, à l'origine, puis les corps de métier, ou les "Symboles" des arts ou des sciences qui en sont dérivés. 
cette cognée oubliés que le Calender et sa maîtresse doivent tous les malheurs qu'expose le texte ${ }^{23}$. Sous couvert de motifs rappelant les symboles et trophées, la vignette décorative investit donc la fonction et l'espace impartis aux vignettes dans le texte (fig.4).

La souplesse de réemploi des gravures, que favorise l'évolution des techniques, et en particulier l'invention du cliché ${ }^{24}$, suppose toutes sortes d'autres variations. Ainsi, le motif du petit chat, qui apparaît dans la narration, peut-il être repris en cul-de-lampe avec une fonction décorative et de ponctuation - marquant joliment la fin du chapitre ${ }^{25}$. Certains bandeaux particulièrement détachés du contexte doivent à cette fonction purement décorative leur réemploi fréquent ; ce sont des bandeaux "omnibus", pour reprendre une idée introduite par Champfleury dans Les Vignettes romantiques (1883). Prenons l'exemple, interchangeable à loisir, de cette femme alanguie sous des rinceaux de feuilles d'acanthe ${ }^{26}$ (fig.5) : femme et fleurs confondues restent sans rapport aucun avec le texte, qui fait allusion à la libération de tout un peuple métamorphosé en poissons ${ }^{27}$. Le contexte du réemploi n'est pas davantage opportun. Et que dire des deux anges coiffés de turbans, encadrant un livre ouvert, et qui n'ont aucun rapport avec le portefaix, sollicité pour le marché d'une belle dame de Bagdad, dont parle le texte ? Pourtant, le livre ouvert est signe : outre qu'il s'agit d'un motif dont les illustrateurs romantiques usent volontiers dans la mesure où ils exploitent constamment les procédés de mise en abîme, il remplit ici son office

${ }^{23}$ Que n'a-til profité de sa bonne fortune sans tenter le diable, ce calender ! : ayant rencontré, dans un souterrain, une belle dame gardée par un démon aux visites épisodiques, il refuse d'admettre ce partage, défie le monstre, mais prend la fuite à son apparition, oubliant les deux indices qui mettront le démon sur sa trace. Tout se termine fort mal : le démon met à mort la belle infidèle et transforme notre héros en singe.

24 Qui permet de multiplier les exemplaires de la matrice gravée d'une vignette, et de substituer le métal à la plaque de buis : les tirages s'en trouvent plus importants, et les modalités de réemploi décuplées, d'autant plus que les fondeurs disposent au cours de la Monarchie de Juillet de catalogues de vignettes vendues à prix marqué.

${ }^{25}$ Le chat apparaît en vignette p. 169 du t. I. Son rôle est alors capital, car les poils de sa queue, dûment prélevés et brûlés, délivreront une princesse d'un sortilège. Le réemploi en cul-de-lampe se trouve p. 59.

${ }^{26}$ Première vignette de cette série de réemplois : t. I, p. 102. Autre exemple p. 277.

${ }^{27}$ Il s'agit d'une magicienne levant son enchantement contre les sujets d'un jeune roi, que dans un esprit de vengeance elle avait réduits à ce peu enviable état (p. 102 et suivantes). 
d'introduction (fig.6) ${ }^{28}$. Le bandeau traduit donc, dans le registre décoratif, la dévotion attentive qui entoure le surgissement du livre.

Toutes ces variations, ces jeux sur le lieu et la fonction, font apparaître un espace de référence lié à la métaphore du livre comme architecture, auquel se superpose l'usage de la vignette, qui introduit l'ordre végétal des vrilles de la vigne et le mouvement décoratif et centrifuge de la spirale tournoyante, sans brouiller complètement l'ordre précédent. Peut-être est-ce à ce jeu sur un double système que l'on doit la vitalité de l'illustration romantique, qui est elle-même de nature organique, fluide, attirée par les métamorphoses, les hybrides et les formes qui passent d'un état dans un autre, plus que par la représentation des états stables, propres au mode architectural classique et néo-classique ${ }^{29}$. Ce glissement de l'illustratif dans le décoratif, cette incursion de la poussée végétale dans l'ordre architectural induisent la catégorie du pittoresque, essentielle à toute l'esthétique romantique, depuis les réflexions sur l'art des jardins anglais de la fin du XVIIIè siècle jusqu'à la conception de la lithographie dans les Voyages pittoresques, où le végétal ronge les formes et détruit l'architecture qui devient ruine. C'est par la catégorie du pittoresque qui glisse et va partout que s'opère en fin de compte la fusion de l'ornemental et de l'illustratif dans le livre romantique.

\section{Images cachées dans les formes récurrentes}

La rhétorique du bandeau obéit à quelques lois simples, qui rejoignent celles de l'art de "la grotesque" analysé par André Chastel dans son dernier livre : c'est en premier lieu une loi de symétrie axiale, qui développe un champ pictural en miroir de part et d'autre de l'axe central, comme dans le schéma corporel humain (que l'on retrouve aux origines des conceptions palladiennes de l'architecture) ; mais une autre loi, celle du caprice ou de la fantaisie, vient, en un endroit quelconque, pervertir l'arrangement stable de la symétrie. Ainsi, dans le bandeau à la femme alanguie (fig.5), le motif humain, qui s'inscrit une seule fois sur la gauche, rompt à peine la symétrie du décor : l'ovale du visage s'inscrit dans les feuillages, tandis qu'une palmette gréco-

28 Cette gravure intervient à un moment-clé du récit, celui où la conteuse prend un risque majeur : celui d'interrompre une histoire avec la venue du jour, au lieu de ménager comme d'habitude un rebondissement. Le livre ouvert symbolise donc le caractère inaugural d'un conte tout neuf, coïncidant avec la coupure de la "nuit" (c'est-à-dire du chapitre).

${ }^{29}$ C'est dans l'oeuvre de l'illustrateur Grandville (qui se fit connaître par Les métamorphoses du jour) que ces tendances se font le mieux sentir. 
romaine se déploie, sans nécessité autre que rythmique, au centre de la composition. L'asymétrie, ludique et capricieuse, à la frange de l'anecdotique qui est son inclination naturelle, ne prend son sens que par rapport à la symétrie qui est de rigueur.

Si la symétrie est la première "loi" - si habilement contournée - du bandeau, la seconde est, pour l'illustration des Mille et Une Nuits, la récurrence du motif de l'arc. La fonction introductive du bandeau est en effet souvent assurée par un arc, suivant la métaphore architecturale fondatrice du porche permettant l'accès à la lecture. Mais de quel déploiement d'ingéniosité vont faire preuve les graveurs, s'efforçant de faire apparaître des arcs sous les objets réels les plus inaptes à cette fonction ! Tout l'art consiste ici à établir un lien entre une forme géométrique, la portion de cercle, et un élément anecdotique : ici, un pont, sur lequel passent des groupes d'hommes et d'éléphants obéissant à la fois à des motivations narratives ${ }^{30}$ et aux lois de la symétrie latérale par rapport à un axe central (fig. 7). D'autres exemples d'actualisations thématiques montrent d'autres ruses de l'arcature : si le motif du pont reste le favori ${ }^{31}$, on rencontre aussi des rideaux s'entrouvrant sur le chapitre $^{32}$, le dôme rond d'un monument ${ }^{33}$, une porte ${ }^{34}$, des arbres mêlant leurs frondaisons ou s'incurvant ${ }^{35}$, le reflet de la lumière dans l'eau ${ }^{36}$, ou tout simplement rien. Rien, si ce n'est la pure idée de l'arche, réduite à l'abstraction de la ligne : quand toute ruse est décidément impossible, le bandeau lui-même s'incurve, rappelant la courbe de l'arc ${ }^{37}$. Il est même des arcs qui sont tout simplement des arcs : trilobé à la mode réputée orientale, le bandeau représentant des hommes brandissant des sabres est tout à la fois un rappel de la figure emblématique de la menace, récurrente dans le texte ${ }^{38}$, et un hommage aux lois de symétrie et d'arcature qui président au bandeau architectural.

Le motif trilobé vaut ici pour lui-même, alors que dans le bandeau aux éléphants, il est dissimulé ${ }^{39}$. Projetés en ombre chinoise - en "ombre portée"40

${ }^{30}$ En conformité avec le texte qui suit : il est question de Bedreddin, qui vend à un juif la cargaison de son navire. Libre au lecteur d'imaginer que les éléphants, sur le pont, transportent ces richesses.

31 Voir par exemple p. 362 et p. 364, t. I.

32 Par exemple p. 135 , p. 240 et p. 290 , t. I.

33 Par exemple p. 188 , t. I.

${ }^{34}$ Par exemple p. 192, p. 227, t. I.

35 Par exemple p. 232, p. 315, t. I.

36 Par exemple p. 222, t. I.

${ }^{37}$ Par exemple p. 128 , p. 168 , p. 203 , p. 474 du t. I

38 Voir note 4.

${ }^{39}$ Il est tentant ici d'en faire appel à la notion de "crypto-image" introduite par Jean-Didier Urbain, dans Eidos, 1992. 
- les éléphants chargés d'un fardeau rond font apparaître le même motif trilobé (fig. 7). Le bandeau, par l' effet de l'image cachée dans l'image, exploite les ressources de la métaphore visuelle ${ }^{41}$, qui, à partir d'une forme donnée, laisse à l'imagination la fantaisie de découvrir tour à tour des figures successives. Ce plaisir du "caprice" retrouve l'une des grandes ressources projectives de la "grotesque", telle qu'André Chastel en a évoqué le mécanisme divertissant. L'ornement laisse à l'imagination libre cours, en lisière du texte, et en toute indépendance capricieuse. L'érotisme dispensé dans les bandeaux des Nuits use souvent de cette ressource de la "cryptoimage", dans laquelle le lecteur est invité, comme dans les figures composées d'Arcimboldo, à découvrir, par-delà une réunion de motifs anodins, une toute autre figure, et par exemple l'anatomie d'un sexe féminin ${ }^{42}$.

La préséance de l'illustration "littérale" sur l'ornement n'empêche pas celui-ci de se déployer dans les espaces qui lui sont dévolus, bandeau et culde-lampe en particulier, en relation avec une autre famille d'objets que le livre, celle des arts décoratifs : le papier peint, les impressions sur étoffes ${ }^{43}$ ou encore les "macédoines" 44 procèdent de la même répétition de motifs disséminés ou regroupés, en dépit de tout ordre narratif linéaire, et de tout sens de lecture prescrit, que l'ornement ludique du bandeau ou du cul-delampe. Entre ces "sujets", pousse le lien facétieux des guirlandes et des

40 Le mot ne vient pas ici par hasard : le caricaturiste Grandville, dans les premiers numéros du journal La Caricature, fondateur d'un langage pour toute l'illustration romantique, avait publié une planche en deux parties - à la manière d'un feuilleton - les Ombres portées, dont tout le sel consistait, par le jeu de l'ombre portée, à métamorphoser les silhouettes des personnages-cibles en objets divers, tels qu'un éteignoir pour le jésuite, un croissant de lune pour le romantique, une cruche pour le moine en froc.... (La Caricature, pl. 3 et 5, $\mathrm{n}^{\circ} 2$ et 3,11 et 18 novembre 1830 ).

${ }^{41}$ C'est là un procédé que le livre illustré des années 1840 emprunte à la caricature des années 1830-35, celui de la métaphore visuelle, décrit par Kris et Gombrich : qu'on pense tout simplement à la célèbre "poire" de Louis-Philippe, résultant de l'identification, au terme de successives métamorphoses, du visage du roi avec la forme projetée du fruit, telle que Philipon la dessina, en quatre "croquades" en cour d'assises pour assurer les jurés de l'innocence de la métamorphose...

42 Ainsi, le bandeau de la page 240, tome I.

43 En particulier celles qui comportent des motifs figurés, des "sujets", comme les toiles de Jouy ou d'Oberkampf.

44 Planches d'imagerie réunissant une variété de "sujets" faits pour être découpés et décorer des boîtes, des écrans ou des paravents, et destinés aux travaux d'agrément des dames du monde ou des enfants. 
feuillages, comme dans la grotesque et l'art du bandeau, qu'en dernier ressort il convient peut-être de réinsérer moins dans la tradition du livre illustré que dans celle des arts décoratifs, où l'ornement se conjugue à la fantaisie, incitant le spectateur à fabuler à son tour à partir des images dépourvues de séquence narrative.

(Université de Reims et CNRS, musée d'Orsay).

Anne Larue

8, rue Geoffroy Saint Hilaire, 75005 Paris.

Ségolène Le Men

10 , rue Royale,

75008 Paris. 